\title{
Warping Entre Imagens e Sua Aplicação à Visualização de Superfícies Topográficas ${ }^{1}$
}

F.S. de SOUSA ${ }^{2}$, M. MENEGUETTE Jr., Departamento de Matemática, Faculdade de Ciências e Tecnologia, UNESP, Cx.P. 957, 19060-900 Presidente Prudente, SP, Brasil.

Resumo. Quando se trabalha com representação de terrenos no computador, busca-se obter modelos mais realísticos do mesmo. Nesse sentido, este trabalho utiliza uma técnica de distorção de imagens, chamada warping por triangulação, aplicando-a à visualização tridimensional de superfícies topográficas, distorcendo a imagem, geralmente provida de fotos de satélite, em cima de seu respectivo modelo digital de terreno.

\section{Introdução}

No intuito de implementar uma ferramenta que fosse capaz de distorcer uma imagem digital, geralmente provida de fotos de satélite, sobre o modelo digital do terreno correspondente, foi estudada uma técnica bastante empregada em recursos de warping e morphing ${ }^{4}$ entre imagens. Antes, porém, é necessária uma certa conceituação teórica sobre o problema e sua descrição, levando-se em conta seus modelos matemáticos, visando uma resolução mais precisa e correta.

\section{Modelo matemático de imagem}

Para representar e manipular imagens no computador, deve-se definir modelos matemáticos adequados a esses objetivos. Quando se observa uma fotografia ou uma cena real, recebe-se de cada ponto um impulso luminoso, que associa uma informação de cor a esse ponto. Dessa forma, o modelo matemático mais natural para descrever uma imagem é o de uma função definida numa superfície bidimensional e tomando valores em um espaço de cor.

Definição 1 Uma imagem é uma aplicação $f: U \subset \mathbb{R}^{2} \rightarrow \mathcal{C}$, onde $\mathcal{C}$ é um espaço de cor.

\footnotetext{
${ }^{1}$ Apoio financeiro da FAPESP

${ }^{2}$ fsimeoni@icmc.sc.usp.br

${ }^{3}$ messias@prudente.unesp.br

${ }^{4}$ Morphing (metamorfose): termo técnico que designa efeito de transição entre imagens.
} 
A função $f$ é chamada de imagem, o conjunto $U$ de suporte da imagem e o conjunto $f(U) \subset \mathcal{C}$ de conjunto de cores da imagem. Em geral, tem-se $\mathcal{C}=\mathbb{R}^{n}$, mas os casos mais comuns são quando $n=3$ e $n=1$. Quando $n=3$, obtém-se um espaço de cores tricromático com base de primárias $R, G$ e $B$, resultando em uma imagem colorida. Quando $n=1$, dizemos que a imagem é monocromática.

A representação mais comum de uma imagem consiste em tomar um subconjunto discreto $U^{\prime} \subset U$ do domínio da função imagem. Neste caso, cada ponto $\left(x_{i}, y_{j}\right) \in U^{\prime}$ é chamado de elemento da imagem ou pixel. O caso mais comum de discretização de uma imagem consiste em tomar o domínio como sendo o retângulo

$$
U=[a, b] \times[c, d]=\left\{(x, y) \in \mathbb{R}^{2}: a \leq x \leq b \text { e } c \leq y \leq d\right\}
$$

e discretizar esse retângulo usando os pontos de um reticulado bidimensional $\Delta=$ $\left(\Delta_{x}, \Delta_{y}\right)$

$$
\Delta=\left\{\left(x_{i}, y_{j}\right) \in U: x_{i}=i \cdot \Delta_{x}, y_{j}=j \cdot \Delta_{y}, i, j \in \mathbb{Z}, \Delta_{x}, \Delta_{y} \in \mathbb{R}\right\} .
$$

Assim, cada pixel $\left(x_{i}, y_{j}\right)$ da imagem pode ser representado por coordenadas inteiras $(i, j)$. Portanto, a imagem pode ser representada de forma conveniente no formato matricial. Nessa representação, a imagem está associada a uma matriz $A$ de dimensão $m \times n$ da seguinte forma:

$$
\begin{aligned}
& A=\left[\begin{array}{cccc}
a_{11} & a_{12} & \ldots & a_{1 n} \\
a_{21} & a_{22} & \cdots & a_{2 n} \\
\vdots & \vdots & \ddots & \vdots \\
a_{m 1} & a_{m 2} & \cdots & a_{m n}
\end{array}\right]=\left[\begin{array}{cccc}
f\left(x_{1}, y_{1}\right) & f\left(x_{1}, y_{2}\right) & \cdots & f\left(x_{1}, y_{n}\right) \\
f\left(x_{2}, y_{1}\right) & f\left(x_{2}, y_{2}\right) & \cdots & f\left(x_{2}, y_{n}\right) \\
\vdots & \vdots & \ddots & \vdots \\
f\left(x_{m}, y_{1}\right) & f\left(x_{m}, y_{2}\right) & \cdots & f\left(x_{m}, y_{n}\right) .
\end{array}\right]
\end{aligned}
$$

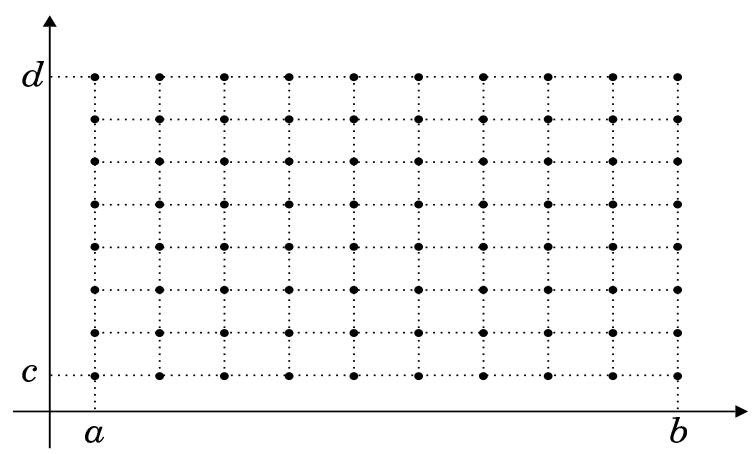

Figura 1: Reticulado uniforme da representação matricial da imagem.

\section{Filtro de warping}

Como visto anteriormente, a operação (ou filtro) que realiza deformações geométricas em uma imagem digital é comumente conhecido, na literatura de processamento de 
imagens, por warping. Seguindo o modelo matemático de imagem dado anteriormente, pode-se definir um filtro de warping como sendo simplesmente uma aplicação $h: U \subset \mathbb{R}^{2} \rightarrow V \subset \mathbb{R}^{2}$.
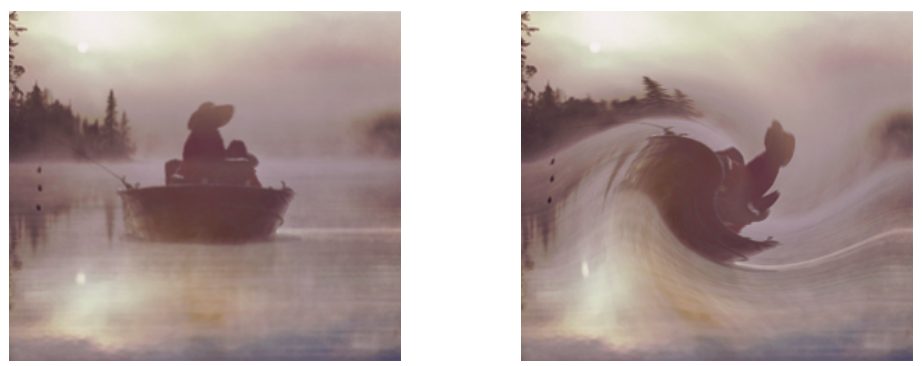

Figura 2: Exemplo de warping em uma imagem.

Em geral, são feitas algumas restrições para a função $h$ que define o warping. Do ponto de vista matemático, é natural exigir que essa função seja uma bijeção e, além disso, tanto $h$ quanto sua inversa $h^{-1}$ sejam contínuas. Uma função com essas características é chamada de homeomorfismo. Neste caso, a injetividade garante que não há superposição de pontos na deformação e a continuidade garante que não haverá rupturas da imagem. Os casos mais interessantes de warping ocorrem quando $h$ é um difeomorfismo, ou seja, $h$ e $h^{-1}$ são de classe $C^{k}$.

A imagem filtrada é obtida por $g=f \circ h^{-1}: U \subset \mathbb{R}^{2} \rightarrow \mathcal{C}$, ilustrado no diagrama abaixo.

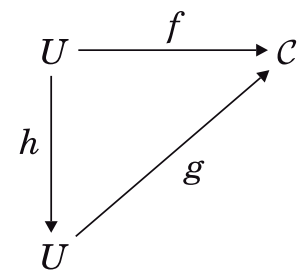

Desse modo, a operação de warping faz uma mudança das coordenadas $(x, y)$ dos pixels da imagem, para um outro sistema de coordenadas $(u, v)$, tal que

$$
u=u(x, y), v=v(x, y) .
$$

Um exemplo simples de warping é dado pela rotação da imagem por um ângulo de $90^{\circ}$ em torno do pixel localizado na origem. Nesse caso, $h$ é um difeomorfismo dado por

$$
h(x, y)=\left[\begin{array}{cc}
\cos 90^{\circ} & -\operatorname{sen} 90^{\circ} \\
\operatorname{sen} 90^{\circ} & \cos 90^{\circ}
\end{array}\right]\left[\begin{array}{l}
x \\
y
\end{array}\right]=\left[\begin{array}{cc}
0 & -1 \\
1 & 0
\end{array}\right]\left[\begin{array}{l}
x \\
y
\end{array}\right]=(-y, x) .
$$




\section{Warping por triangulação}

A maneira mais simples de se aplicar um filtro de warping é definir um sistema de coordenadas para a imagem através de uma malha de pontos, chamada de máscara.

Definição 2 Seja $f: U \subset \mathbb{R}^{2} \rightarrow \mathcal{C}$ uma imagem. Uma máscara para $f$ é uma matriz $M$ de dimensão $m \times n$ cujas entradas são pontos de $U$.

Tendo em vista a máscara $M$, podemos obter uma triangulação da mesma tomando triângulos da forma $\left(M_{i j}, M_{i j+1}, M_{i+1 j+1}\right)$ e $\left(M_{i j}, M_{i+1 j}, M_{i+1 j+1}\right)$, evitando assim uma triangulação de Delaunay, diminuindo a complexidade do problema.

A transformação de warping de uma imagem $f$ será obtida a partir de uma transição entre duas máscaras distintas de $f$, mas com a mesma ordem. Assim, dados $M$ e $N$ duas máscaras distintas de $f, A$ e $B$ triângulos de $M$ e $N$, respectivamente, sabemos que existe uma única transformação afim $T: U \rightarrow \mathbb{R}^{2}$ que transforma o triângulo $A$ no triângulo $B$, ou seja, $T(A)=B$. É possível, então, construir uma função contínua $h$ que associa a cada triângulo da máscara $M$, o triângulo correspondente (de mesmos índices) na máscara $N$, bastando definir a restrição da função $h$ naquele triângulo como sendo a transformação afim associada. Matematicamente, a função de warping será dada por:

$$
h: U \subset \mathbb{R}^{2} \rightarrow \mathbb{R}^{2},\left.\operatorname{com} h\right|_{A_{i}}=T_{i}, \forall A_{i} \in M
$$

onde $T_{i}$ é a única transformação afim entre os triângulos correspondentes $A_{i}$ e $B_{i}$ tal que $T_{i}\left(A_{i}\right)=B_{i}$.
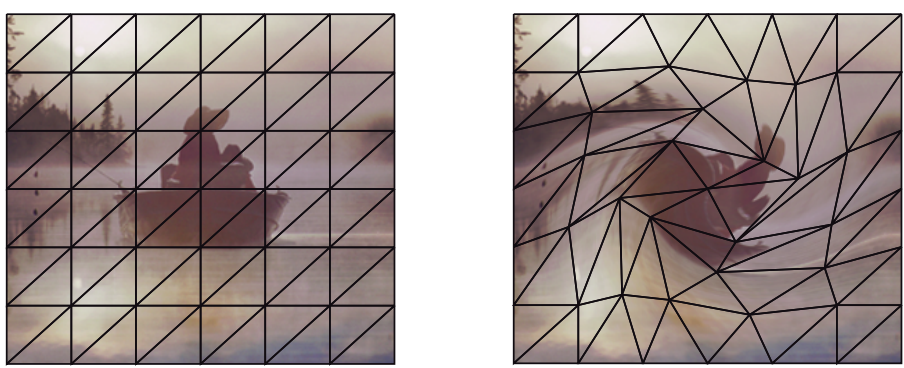

Figura 3: Warping de uma imagem com sua máscara.

\subsection{Algoritmo para warping por triangulação}

Com tudo o que foi visto até agora, pode-se definir um algoritmo que, dado uma imagem e suas máscaras inicial e final, encontre a transformação de warping $h$, aplicando-a na mesma. Tal algoritmo pode ser descrito através do fluxograma da Figura 4. 


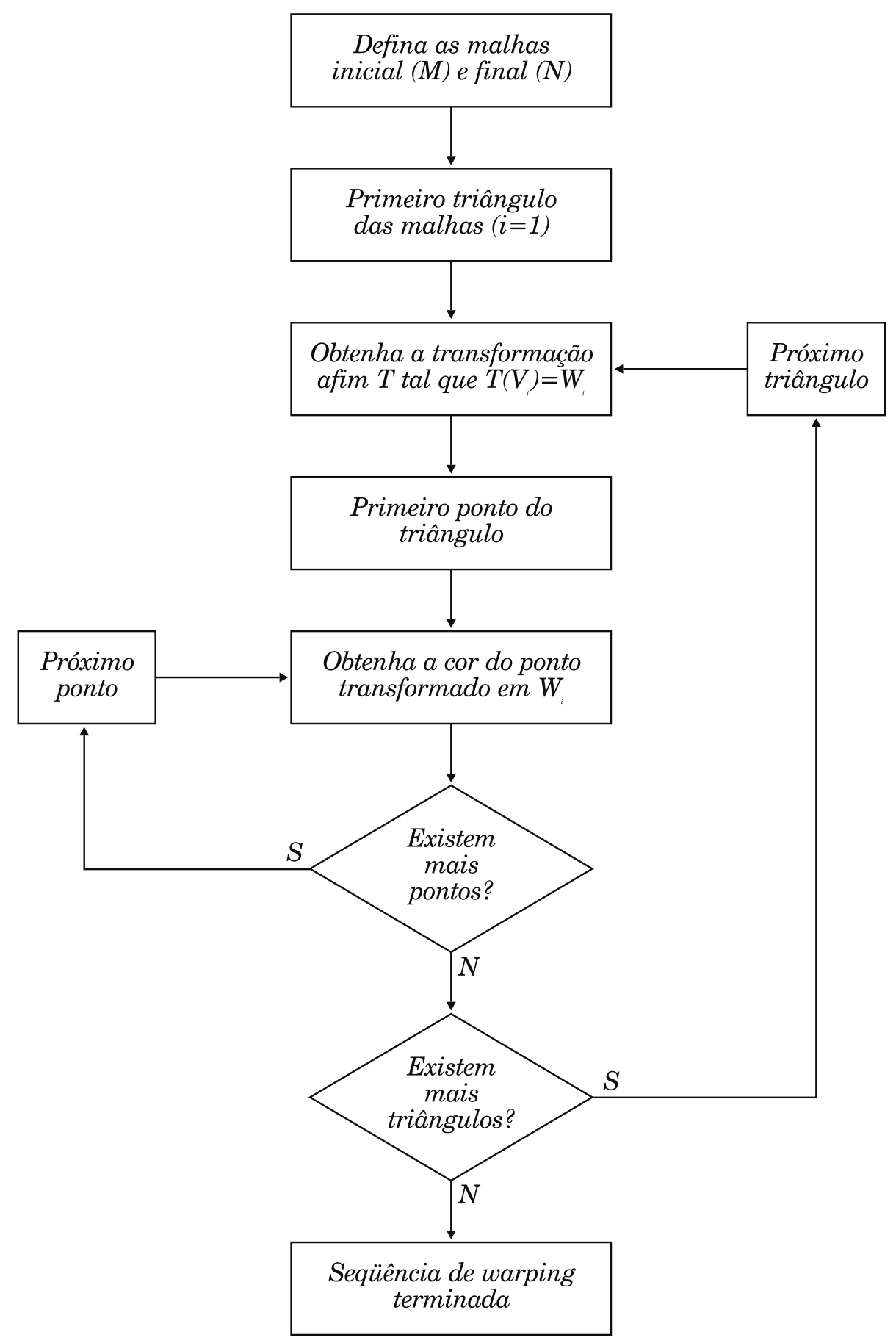

Figura 4: Fluxograma para o algoritmo de warping por triangulação 
Porém, a implementação desta técnica de warping traz alguns problemas como, por exemplo, encontrar os pontos da imagem que pertencem a um triângulo da máscara, ou ainda, a determinação de cada transformação $T$ que mapeia os triângulos correspondentes nas máscaras $M$ e $N$. Como resultado do estudo desta técnica, apresenta-se, a seguir, possíveis soluções para estes problemas.

\section{2. $\quad$ O problema de pontos interiores}

O problema da determinação dos pontos interiores de um triângulo pode ser apresentado da seguinte forma: "Dado um triângulo com vértices $A, B$ e $C$ e um ponto $D$, determinar se $D$ pertence ao interior do triângulo $A B C$ ".

Para a solução deste problema, podemos enunciar um teorema cuja demonstração é trivial e não será feita aqui.

Teorema 1 Sejam $A=\left(x_{a}, y_{a}\right), B=\left(x_{b}, y_{b}\right)$ e $C=\left(x_{c}, y_{c}\right)$ os vértices de um triângulo e $D=\left(x_{d}, y_{d}\right)$ um ponto qualquer. $O$ ponto $D$ pertence ao interior do triângulo $A B C$ se, e somente se, pertencer ao interior dos ângulos $C A B$ e $A B C$.
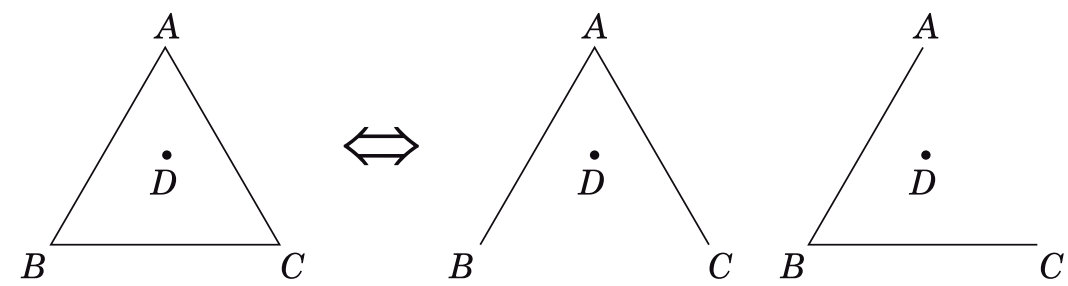

Tendo o resultado do Teorema 1, basta então obter um algoritmo que determine se o ponto dado pertence ao interior de um determinado ângulo. Para tanto, sejam $\bar{A}=\left(x_{a}, y_{a}, 0\right), \bar{B}=\left(x_{b}, y_{b}, 0\right), \bar{C}=\left(x_{c}, y_{c}, 0\right)$ e $\bar{D}=\left(x_{d}, y_{d}, 0\right)$. Definindo os vetores $u=\bar{B}-\bar{A}, v=\bar{C}-\bar{A}$ e $w=\bar{D}-\bar{A}$, o ponto $D$ está no interior do ângulo $C A B$ se, e somente se, as bases $\{u, v, u \times w\}$ e $\{u, v, v \times w\}$ tem orientações contrárias $^{5}$. De fato, temos duas possíveis situações para o vetor $w$ :

$1^{\circ}$ Caso: $\mathrm{O}$ vetor $w$ pertence ao interior do ângulo formado pelos vetores $u$ e $v$. Graficamente, esta situação pode ser ilustrada pelo seguinte gráfico:

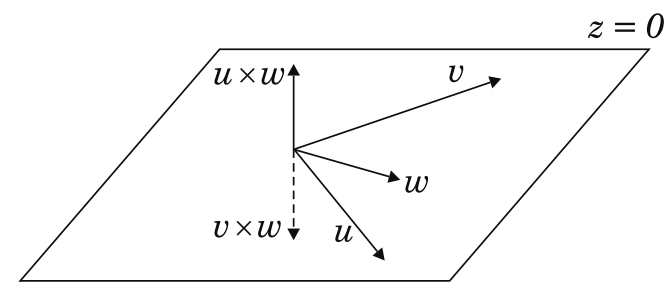

É fácil perceber que os vetores $u \times w$ e $v \times w$ terão mesma direção, mas sentidos opostos.

\footnotetext{
${ }^{5}$ Aqui, $u \times v$ denota o produto vetorial de $u$ por $v$.
} 
$\mathbf{2}^{o}$ Caso: $\mathrm{O}$ vetor $w$ não pertence ao interior do ângulo formado pelos vetores $u \mathrm{e}$ $v$. Graficamente, esta situação pode ser visualizada pelos seguintes gráficos:

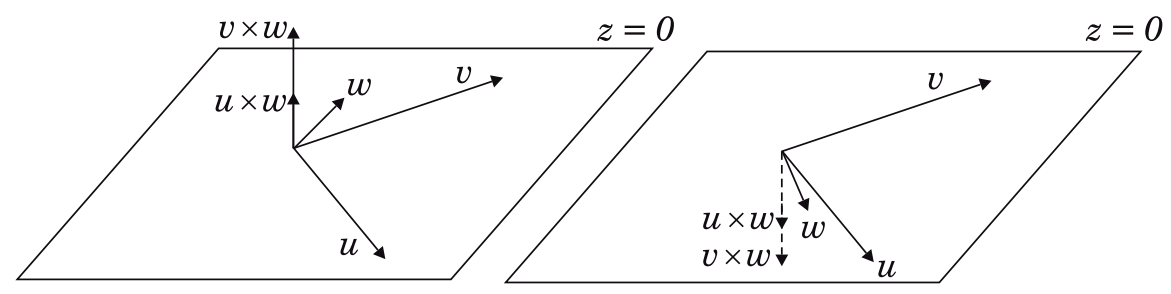

Nesta situação, os vetores $u \times w$ e $v \times w$ terão não apenas a mesma direção, mas também o mesmo sentido. Agora, como

$$
\begin{gathered}
u \times w=\left|\begin{array}{ccc}
\vec{i} & \vec{j} & \vec{k} \\
x_{b}-x_{a} & y_{b}-y_{a} & 0 \\
x_{d}-x_{a} & y_{d}-y_{a} & 0
\end{array}\right|=\left(0,0, u_{w}\right) \\
v \times w=\left|\begin{array}{ccc}
\vec{i} & \vec{j} & \vec{k} \\
x_{c}-x_{a} & y_{c}-y_{a} & 0 \\
x_{d}-x_{a} & y_{d}-y_{a} & 0
\end{array}\right|=\left(0,0, v_{w}\right)
\end{gathered}
$$

onde

$$
\begin{aligned}
& u_{w}=\left(x_{b}-x_{a}\right)\left(y_{d}-y_{a}\right)-\left(y_{b}-y_{a}\right)\left(x_{d}-x_{a}\right) \\
& v_{w}=\left(x_{c}-x_{a}\right)\left(y_{d}-y_{a}\right)-\left(y_{c}-y_{a}\right)\left(x_{d}-x_{a}\right)
\end{aligned}
$$

segue que o ponto $D$ está no interior do ângulo formado por $u$ e $v$ se, e somente se, os números $u_{w}$ e $v_{w}$ têm sinais opostos, ou ainda, se $u_{w} \cdot v_{w} \leq 0$.

Como foi visto na seção 2., uma imagem $f$ pode ser representada por uma matriz de dimensão $m \times n$, e assim, as coordenadas dos pixels da imagem podem ser representados por números inteiros. Na verdade, quando se implementa uma ferramenta de processamento de imagens, cujo objetivo é mostrar o resultado obtido na tela do computador, usa-se coordenadas inteiras, pois os pixels do monitor são referenciados assim.

Desta forma, para verificar se o próximo ponto, digamos $\left(x_{d+1}, y_{d}\right)=\left(x_{d}+1, y_{d}\right)$, observe que

$$
\begin{aligned}
\overline{u_{w}} & =\left(x_{b}-x_{a}\right)\left(y_{d}-y_{a}\right)-\left(y_{b}-y_{a}\right)\left(x_{d}+1-x_{a}\right) \\
& =\left(x_{b}-x_{a}\right)\left(y_{d}-y_{a}\right)-\left(y_{b}-y_{a}\right)\left(x_{d}-x_{a}\right)-\left(y_{b}-y_{a}\right) \\
& =u_{w}-\left(y_{b}-y_{a}\right)
\end{aligned}
$$

Analogamente,

$$
\begin{aligned}
\overline{v_{w}} & =\left(x_{c}-x_{a}\right)\left(y_{d}-y_{a}\right)-\left(y_{c}-y_{a}\right)\left(x_{d}+1-x_{a}\right) \\
& =\left(x_{c}-x_{a}\right)\left(y_{d}-y_{a}\right)-\left(y_{c}-y_{a}\right)\left(x_{d}-x_{a}\right)-\left(y_{c}-y_{a}\right) \\
& =v_{w}-\left(y_{c}-y_{a}\right)
\end{aligned}
$$


Assim, como os fatores $A_{1}=\left(y_{b}-y_{a}\right)$ e $A_{2}=\left(y_{c}-y_{a}\right)$ dependem apenas do triângulo dado e são constantes, o cálculo do produto vetorial é realizado apenas no início, bastando somar estas constantes para os demais pontos da linha. De modo análogo ao que foi feito em (4.2) e (4.3), para verificar se o ponto $\left(x_{d}, y_{d+1}\right)=$ $\left(x_{d}, y_{d}+1\right)$ pertence ao ângulo, basta notar que

$$
\begin{aligned}
\widetilde{u_{w}} & =u_{w}+\left(x_{b}-x_{a}\right), \\
\widetilde{v_{w}} & =v_{w}+\left(x_{c}-x_{a}\right) .
\end{aligned}
$$

Desta maneira, uma vez obtidos os valores iniciais de $u_{w}$ e $v_{w}$, basta acrescentar valores inteiros aos mesmos para obter os valores de $u_{w}$ e $v_{w}$ para o próximo ponto. Isto torna o algoritmo extremamente rápido e eficiente, melhorando sobremaneira a eficiência do processo de warping.

\subsection{Determinação das transformações}

Como visto em (4.1), o filtro de warping por triangulação pode ser dado por uma função $h$ tal que a sua restrição a um triângulo da malha $M$ mapeia-o em seu triângulo correspondente na malha $N$, através de uma transformação afim.

O problema proposto aqui é o seguinte: "Dados dois triângulos $V, W \subset \mathbb{R}^{2}$, encontrar a única transformação afim $T: \mathbb{R}^{2} \rightarrow \mathbb{R}^{2}$ tal que $T(V)=W$."

De uma forma mais específica, sendo $p_{1}, p_{2}$ e $p_{3}$ vértices de $V$ e $q_{1}, q_{2}$ e $q_{3}$ vértices de $W$, tais que

$$
\begin{array}{ll}
p_{1}=\left(v_{1}, w_{1}\right), & q_{1}=\left(x_{1}, y_{1}\right), \\
p_{2}=\left(v_{2}, w_{2}\right), & q_{2}=\left(x_{2}, y_{2}\right), \\
p_{3}=\left(v_{3}, w_{3}\right), & q_{3}=\left(x_{3}, y_{3}\right),
\end{array}
$$

encontrar a transformação $T$ tal que $T(V)=W$ é equivalente a encontrar uma transformação $T$ tal que

$$
\begin{aligned}
& T\left(p_{1}\right)=q_{1}, \\
& T\left(p_{2}\right)=q_{2}, \\
& T\left(p_{3}\right)=q_{3} .
\end{aligned}
$$

Antes de determinar quem é $T$, é necessário ter em mãos alguns resultados que são enunciados a seguir:

Teorema 2 Sejam $p_{1}, p_{2}$ e $p_{3}$ pontos não colineares de $\mathbb{R}^{2}$. Então, cada ponto $p$ do plano pode ser escrito de modo único na forma

$$
p=\lambda_{1} p_{1}+\lambda_{2} p_{2}+\lambda_{3} p_{3}
$$

onde $\lambda_{1}, \lambda_{2}$ e $\lambda_{3}$ são números reais satisfazendo $\lambda_{1}+\lambda_{2}+\lambda_{3}=1$. Os coeficientes $\lambda_{1}, \lambda_{2}$ e $\lambda_{3}$ são denominados coordenadas baricêntricas de $p$ em relação a $p_{1}, p_{2} e$ $p_{3}$. 
Demonstração: Basta observar que, dados $p, p_{1}, p_{2}$ e $p_{3}$, sendo $p=(x, y)$ e $p_{i}=$ $\left(x_{i}, y_{i}\right), i=1,2,3$, os termos $\lambda_{1}, \lambda_{2}$ e $\lambda_{3}$, tais que $\sum_{i=1}^{3} \lambda_{i}=1$, são soluções do seguinte sistema de três equações a três incógnitas:

$$
\left\{\begin{array}{c}
\lambda_{1} x_{1}+\lambda_{2} x_{2}+\lambda_{3} x_{3}=x \\
\lambda_{1} y_{1}+\lambda_{2} y_{2}+\lambda_{3} y_{3}=y \\
\lambda_{1}+\lambda_{2}+\lambda_{3}=1
\end{array}\right.
$$

onde o determinante do sistema é dado por

$$
D=\left|\begin{array}{lll}
x_{1} & x_{2} & x_{3} \\
y_{1} & y_{2} & y_{3} \\
1 & 1 & 1
\end{array}\right|=\left|\begin{array}{llll}
x_{1} & x_{2} & x_{3} & x_{1} \\
y_{1} & y_{2} & y_{3} & y_{1}
\end{array}\right|
$$

que representa o dobro da área do triângulo de vértices $p_{1}, p_{2}$ e $p_{3}$, já que estes não são colineares. Portanto, $D \neq 0$ e o sistema possui solução única para cada $p$, o que demonstra o teorema.

Generalizando este teorema, obtém-se o seguinte resultado:

Corolário 1 Sejam $u_{0}, u_{1}, \ldots, u_{n}, n+1$ pontos do espaço $\mathbb{R}^{n}$, de modo que o conjunto $\left\{u_{1}-u_{0}, u_{2}-u_{0}, \ldots, u_{n}-u_{0}\right\}$ forma uma base deste espaço. Então, qualquer ponto $u \in \mathbb{R}^{n}$ pode ser escrito como uma combinação de suas coordenadas baricêntricas da seguinte forma

$$
u=\sum_{i=0}^{n} \lambda_{i} u_{i}, \operatorname{com} \lambda_{i} \in \mathbb{R}, \sum_{i=0}^{n} \lambda_{i}=1 .
$$

Definição 3 Uma transformação $T: \mathbb{R}^{n} \rightarrow \mathbb{R}^{n}$ é dita afim se

$$
T((1-t) u+t v)=(1-t) T(u)+t T(v), \forall u, v \in \mathbb{R}^{n}, \forall t \in \mathbb{R} .
$$

Teorema 3 Sejam $u=\sum_{i=0}^{n} \lambda_{i} u_{i}$ um vetor do $\mathbb{R}^{n}$ escrito como uma combinação baricêntrica dos vetores $u_{0}, u_{1}, u_{2}, \ldots, u_{n}$, e $T: \mathbb{R}^{n} \rightarrow \mathbb{R}^{n}$ uma transformação afim. Então

$$
T(u)=T\left(\sum_{i=0}^{n} \lambda_{i} u_{i}\right)=\sum_{i=0}^{n} \lambda_{i} T\left(u_{i}\right) .
$$

Demonstração: Será feita por indução finita. Para o caso $n=1$, temos $\lambda_{0}+\lambda_{1}=$ $1 \Rightarrow \lambda_{1}=1-\lambda_{0}$. Daí, pela definição de transformação afim, vem

$$
\begin{aligned}
T\left(\lambda_{0} u+\lambda_{1} v\right) & =T\left(\lambda_{0} u+\left(1-\lambda_{0}\right) v\right) \\
& =\lambda_{0} T(u)+\left(1-\lambda_{0}\right) T(v) \\
& =\lambda_{0} T(u)+\lambda_{1} T(v) .
\end{aligned}
$$

Agora, suponha que o teorema seja válido para $n-1$ pontos, isto é, 


$$
T\left(\sum_{i=0}^{n-1} \lambda_{i} u_{i}\right)=\sum_{i=0}^{n-1} \lambda_{i} T\left(u_{i}\right) .
$$

Sejam $\lambda_{0}, \lambda_{1}, \ldots, \lambda_{n} \in \mathbb{R}$ tais que $\sum_{i=0}^{n} \lambda_{i}=1$ e $u=\sum_{i=0}^{n} \lambda_{i} u_{i} \in \mathbb{R}^{n}$. Assim,

$$
\lambda_{0}+\lambda_{1}+\ldots+\lambda_{n}=1 \Rightarrow \lambda_{0}+\lambda_{1}+\ldots+\lambda_{n-1}=1-\lambda_{n} .
$$

Se $\lambda_{n}=0$ então o teorema está provado, pois recai no caso $n-1$. É claro que $\lambda_{n} \neq 1$, pois se fosse, teríamos $\lambda_{1}=\lambda_{2}=\ldots=\lambda_{n-1}=0 \mathrm{e}$, neste caso, eles não formariam uma combinação baricêntrica. Caso contrário,

$$
\lambda_{0}+\lambda_{1}+\ldots+\lambda_{n-1}=1-\lambda_{n} \Rightarrow \frac{\lambda_{0}+\lambda_{1}+\ldots+\lambda_{n-1}}{1-\lambda_{n}}=\sum_{i=0}^{n-1} \frac{\lambda_{i}}{1-\lambda_{n}}=1 .
$$

Daí,

$$
\begin{aligned}
& T\left(\sum_{i=0}^{n} \lambda_{i} u_{i}\right)=T\left(\left(1-\lambda_{n}\right)\left(\frac{\lambda_{0} u_{0}+\lambda_{1} u_{1}+\ldots+\lambda_{n-1} u_{n-1}}{1-\lambda_{n}}\right)+\lambda_{n} u_{n}\right) \\
& \stackrel{(4.8)}{=}\left(1-\lambda_{n}\right) T\left(\frac{\lambda_{0} u_{0}+\lambda_{1} u_{1}+\ldots+\lambda_{n-1} u_{n-1}}{1-\lambda_{n}}\right)+\lambda_{n} T\left(u_{n}\right) \\
& \stackrel{(4.10)}{=}\left(1-\lambda_{n}\right) T\left(\sum_{i=0}^{n-1} \frac{\lambda_{i}}{1-\lambda_{n}} u_{i}\right)+\lambda_{n} T\left(u_{n}\right) \\
& \stackrel{(4.9)}{=}\left(1-\lambda_{n}\right) \sum_{i=0}^{n-1} \frac{\lambda_{i}}{1-\lambda_{n}} T\left(u_{i}\right)+\lambda_{n} T\left(u_{n}\right) \\
& =\lambda_{0} T\left(u_{0}\right)+\lambda_{1} T\left(u_{1}\right)+\ldots+\lambda_{n-1} T\left(u_{n-1}\right)+\lambda_{n} T\left(u_{n}\right) \\
& =\sum_{i=0}^{n} \lambda_{i} T\left(u_{i}\right)
\end{aligned}
$$

como queríamos demonstrar. Logo, o teorema é válido $\forall n \in \mathbb{N}$.

Agora, seja $(v, w)$ um vetor do plano. Podemos escrevê-lo como combinação baricêntrica dos vetores $p_{1}, p_{2}$ e $p_{3}$ dados em (4.4). Mais precisamente,

$$
(v, w)=\lambda_{1}\left(v_{1}, w_{1}\right)+\lambda_{2}\left(v_{2}, w_{2}\right)+\lambda_{3}\left(v_{3}, w_{3}\right), \operatorname{com} \sum_{i=1}^{3} \lambda_{i}=1,
$$

de onde se obtém um sistema análogo ao dado em (4.6), com representação matricial

$$
\left[\begin{array}{ccc}
v_{1} & v_{2} & v_{3} \\
w_{1} & w_{2} & w_{3} \\
1 & 1 & 1
\end{array}\right]\left[\begin{array}{l}
\lambda_{1} \\
\lambda_{2} \\
\lambda_{3}
\end{array}\right]=\left[\begin{array}{l}
v \\
w \\
1
\end{array}\right]
$$


cuja solução é dada por

$$
\left[\begin{array}{l}
\lambda_{1} \\
\lambda_{2} \\
\lambda_{3}
\end{array}\right]=\frac{1}{k} \cdot\left[\begin{array}{c}
\left(v-v_{2}\right)\left(w-w_{3}\right)-\left(v-v_{3}\right)\left(w-w_{2}\right) \\
\left(v-v_{3}\right)\left(w-w_{1}\right)-\left(v-v_{1}\right)\left(w-w_{3}\right) \\
\left(v-v_{1}\right)\left(w-w_{2}\right)-\left(v-v_{2}\right)\left(w-w_{1}\right)
\end{array}\right],
$$

onde $k=\left(v_{1}-v_{2}\right)\left(w_{1}-w_{3}\right)-\left(v_{1}-v_{3}\right)\left(w_{1}-w_{2}\right)$.

Assim, usando o fato de $T$ ser uma transformação afim, pode-se aplicar os resultados obtidos em (4.5) e no Teorema 3 , donde

$$
\begin{aligned}
T(v, w) & =T\left(\lambda_{1}\left(v_{1}, w_{1}\right)+\lambda_{2}\left(v_{2}, w_{2}\right)+\lambda_{3}\left(v_{3}, w_{3}\right)\right) \\
& =\lambda_{1} T\left(v_{1}, w_{1}\right)+\lambda_{2} T\left(v_{2}, w_{2}\right)+\lambda_{3} T\left(v_{3}, w_{3}\right) \\
& =\lambda_{1}\left(x_{1}, y_{1}\right)+\lambda_{2}\left(x_{2}, y_{2}\right)+\lambda_{3}\left(x_{3}, y_{3}\right) \\
& =\left(\lambda_{1} x_{1}+\lambda_{2} x_{2}+\lambda_{3} x_{3}, \lambda_{1} y_{1}+\lambda_{2} y_{2}+\lambda_{3} y_{3}\right),
\end{aligned}
$$

onde os valores $\lambda_{1}, \lambda_{2}$ e $\lambda_{3}$ são dados por (4.13).

Finalmente, para se obter uma aplicação afim que transforma o triângulo $V$ no triângulo $W$, primeiro calcula-se as coordenadas baricêntricas de um ponto $(v, w)$ em relação aos vértices de $V$ e, depois, para encontrar $T(v, w)$, basta escrever os vértices de $W$ como uma combinação das mesmas coordenadas baricêntricas encontradas.

Pode-se observar ainda que, para obter uma forma genérica para $T$, basta fazer

$$
\begin{aligned}
& \lambda_{1}(v, w)=\frac{1}{k}\left(\left(v-v_{2}\right)\left(w-w_{3}\right)-\left(v-v_{3}\right)\left(w-w_{2}\right)\right), \\
& \lambda_{2}(v, w)=\frac{1}{k}\left(\left(v-v_{3}\right)\left(w-w_{1}\right)-\left(v-v_{1}\right)\left(w-w_{3}\right)\right), \\
& \lambda_{3}(v, w)=\frac{1}{k}\left(\left(v-v_{1}\right)\left(w-w_{2}\right)-\left(v-v_{2}\right)\left(w-w_{1}\right)\right),
\end{aligned}
$$

e a transformação afim $T: \mathbb{R}^{2} \rightarrow \mathbb{R}^{2}$ é dada por

$$
T(v, w)=\lambda_{1}(v, w)\left(x_{1}, y_{1}\right)+\lambda_{2}(v, w)\left(x_{2}, y_{2}\right)+\lambda_{3}(v, w)\left(x_{3}, y_{3}\right) .
$$

\subsection{Interpolação dos pontos transformados}

Um dos problemas gerados pela deformação de imagens é a criação de "buracos", devido ao aumento da área da figura transformada. Este buracos nada mais são do que pontos na imagem final $g$ a qual não foram atribuídos valores pela transformação de warping $h$.

A Figura 5 mostra com clareza o efeito causado por este problema. Para resolvêlo, existem várias técnicas de interpolação que podem ser aplicadas, porém, as mais utilizadas são replicação de pixels (também chamado de filtro box) e interpolação bilinear, devido ótima relação entre custo computacional e qualidade dos resultados.

\subsubsection{Técnica de replicação de pixels}

Esta técnica consiste em, durante o processo de aplicação do warping, fazer com que os pixels não definidos na imagem resultante sejam preenchidos com o valor 
do pixel definido mais próximo. Usualmente, este processo se resume em apenas repetir na linha e na coluna seguinte da imagem resultante, o valor do pixel atual. Por exemplo, considere um fragmento da imagem original $f$ dado por

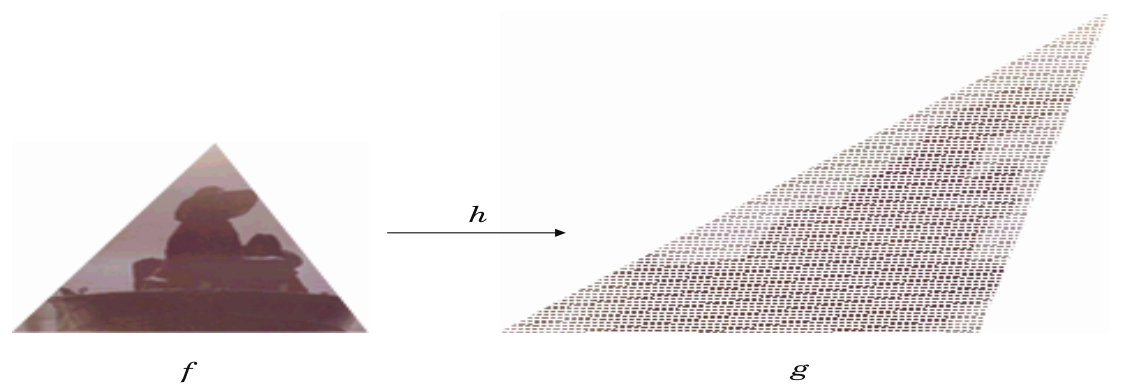

Figura 5: Transformação de warping $h$ aplicado a uma imagem $f$ e a imagem resultante $g$.

$$
\begin{array}{cccc} 
& \vdots & \vdots & \\
\cdots & f(i, j) & f(i, j+1) & \cdots \\
\cdots & f(i+1, j) & f(i+1, j+1) & \cdots \\
& \vdots & \vdots
\end{array}
$$

Após a aplicação da transformação $h$, obtém-se, por exemplo, a imagem $g$ dada por

$$
\begin{array}{ccccc} 
& & \vdots & & \\
& g(i, j) & 0 & g(i, j+1) & \\
\cdots & 0 & 0 & 0 & \cdots \\
& g(i+1, j) & 0 & g(i+1, j+1) & \\
& & \vdots & &
\end{array}
$$

Daí, aplicando-se o filtro descrito, vem

$$
\begin{array}{ccccc} 
& & \vdots & & \\
& g(i, j) & g(i, j) & g(i, j+1) & \\
\cdots & g(i, j) & g(i, j) & g(i, j+1) & \ldots \\
g(i+1, j) & g(i+1, j) & g(i+1, j+1) & \\
& \multicolumn{4}{c}{} \\
& & &
\end{array}
$$

Esse filtro, apesar de ser o mais rápido, é o que possui piores resultados. Como aumentam-se os detalhes da imagem, os defeitos inerentes desta reconstrução ficam mais visíveis, deixando na imagem um efeito "quadriculado". No entanto, esse recurso é bem popular, devido à sua facilidade de implementação e eficiência computacional. 


\subsubsection{Interpolação bilinear}

O método da interpolação bilinear consiste em obter a cor de um pixel não definido através do cálculo de médias, aplicado entre as linhas e colunas da imagem. Para simplificar, considere que os pixel da imagem $g$ são dados por $g(i, j)$. Assim, no mesmo exemplo acima, sendo a imagem após a transformação de warping dada por

$$
\begin{array}{cccc} 
& & \vdots & \\
& g(i, j) & 0 & g(i, j+1) \\
\cdots & 0 & 0 & 0 \\
& g(i+1, j) & 0 & g(i+1, j+1) \\
& & \vdots &
\end{array}
$$

aplicando-se a interpolação bilinear, obtemos

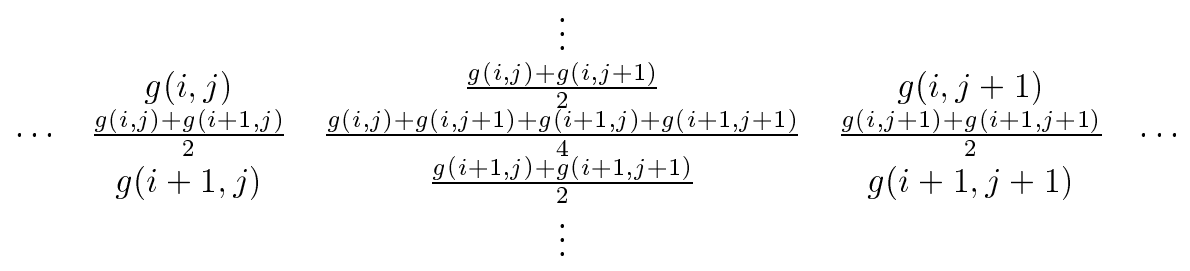

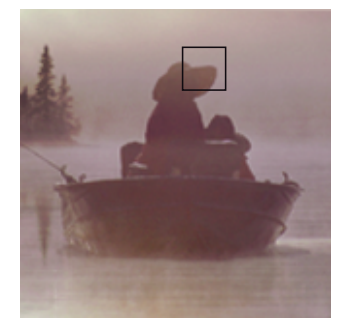

(a)

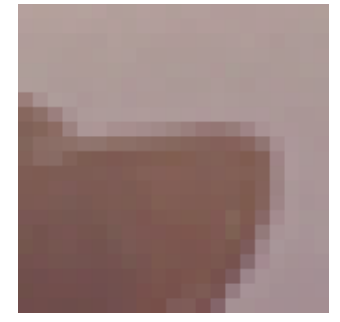

(b)

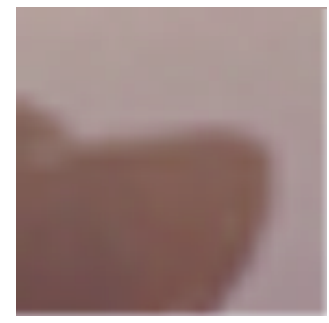

(c)

Figura 6: Transformação de zoom da imagem $(a)$ por $(b)$ replicação de pixels e $(c)$ interpolação bilinear.

A interpolação bilinear apresenta bons resultados e, ainda, é bastante simples na sua implementação, o que o torna um dos métodos mais utilizados na prática. A Figura 6 traz um exemplo onde a aplicação de warping é simplesmente um zoom com escala 2 , onde pode-se constatar na prática a diferença entre estes dois métodos. 


\section{Aplicação de warping à visualização de super- fícies topográficas}

Como aplicação de todo este estudo sobre técnicas de warping, foi implementada uma ferramenta no módulo de visualização de superfícies topográficas MicroDTM já desenvolvido (ver em [7] e [8]), que é capaz de distorcer uma dada imagem em cima de um modelo digital de terreno criado neste programa.

Aproveitando o fato de que o MicroDTM trabalha com uma estrutura de dados organizada na forma de malha triangular, este processo torna-se muito simples. Em primeiro lugar, tem-se uma malha regular tridimensional como modelo digital do terreno, onde cada vértice tem coordenadas $(x, y, z)$. Após a aplicação das rotações e da projeção escolhida, obtém-se uma malha bidimensional, com o mesmo número de triangulos, só que totalmente transformada, o que dá o efeito tridimensional ao terreno.

De posse do número de triângulos da malha e da imagem digital, constrói-se uma malha regular bidimensional em cima nas dimensões da imagem e, cada triângulo desta malha corresponde a somente um triângulo da malha transformada.

Desta forma, basta aplicar a técnica de warping por triangulação em cima destas duas malhas, obtendo a imagem distorcida em cima do modelo do terreno, como era esperado. A plotagem da imagem é feita através de uma adaptação no algoritmo do pintor, que determina a ordem de apresentação dos triângulos e em seguida os preenche com a parte da imagem que lhe corresponde. Todo esse processo pode ser visualizado nas figuras seguintes.
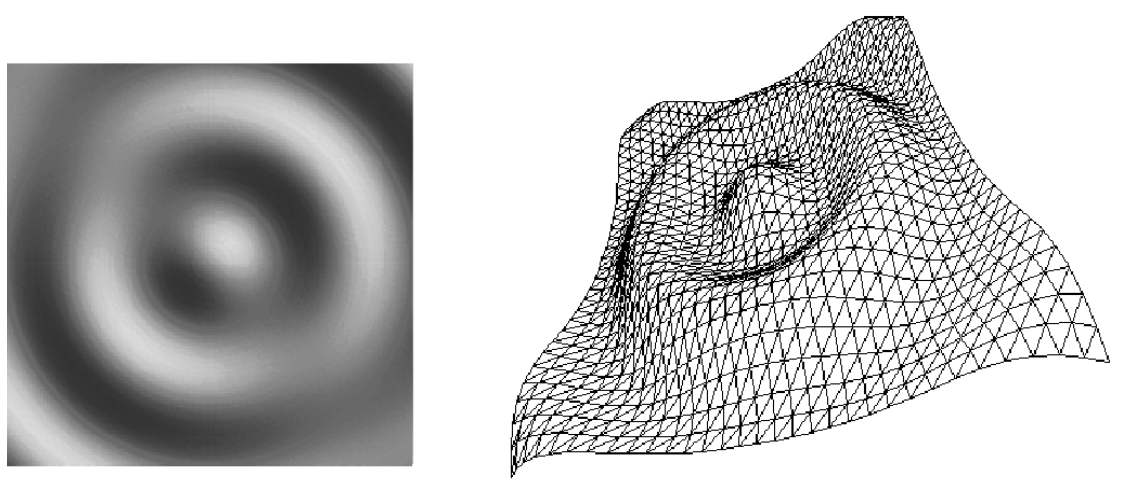

Figura 7: Textura a ser aplicada no gráfico da função $\cos \sqrt{x^{2}+y^{2}}$, obtida no MicroDTM. 


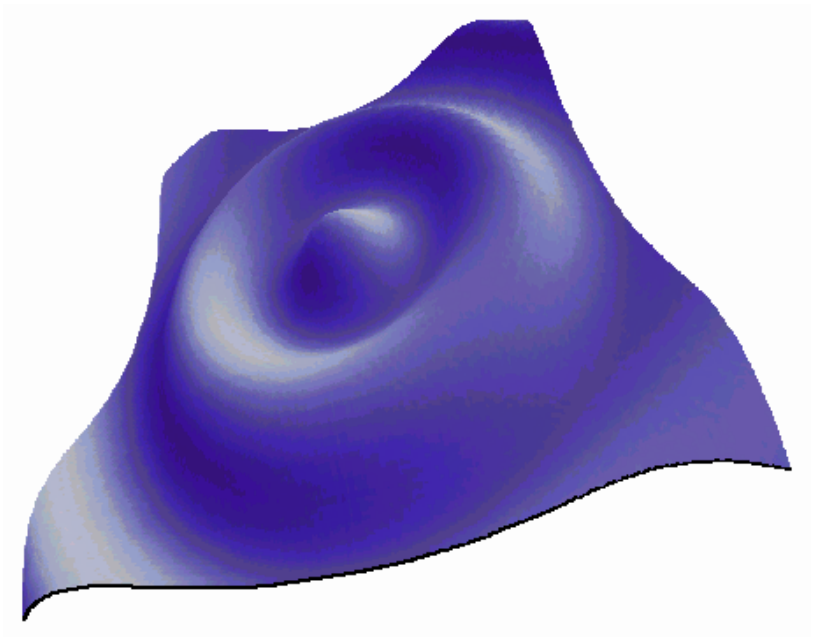

Figura 8: Resultado da distorção, sem a grade da malha triangular.

\section{Referências}

[1] A. O. Artero e M. Meneguette, Transformações Espaciais e Técnicas de Interpolação Aplicadas em Imagens, Colloquium, 1 (3) (1998), 43-51.

[2] E. Doescher e M. Meneguette, Deformações Geométricas em Imagens: metamorfose, registro e texturização, Monografia - FCT/UNESP, 1996.

[3] L. H. Figueiredo e P. C. P. Carvalho, Introdução à Geometria Computacional, $18^{\circ}$ Colóquio Brasileiro de Matemática, IMPA, 1991.

[4] J. Gomes e L. Velho, "Computação Gráfica: Imagem", IMPA, 1994.

[5] J. Gomes e L. Velho, "Computação Gráfica", Volume 1, IMPA, 1998.

[6] E. L. Lima, "Curso de Análise", Volume 2, IMPA, 1981.

[7] F. S. Sousa e M. Meneguette Jr., Apresentação computacional de objetos 3D em vistas paralela e perspectiva, com ênfase a superfícies topográficas e isolinhas, XXI CNMAC, setembro, 1998.

[8] F. S. Sousa e M. Meneguette Jr., MicroDTM: Um módulo para a visualização de superfícies topográficas, I Fórum de Ciências da FCT, novembro, 1998. 
